\title{
Tratamento cirúrgico de fraturas Le Fort I e Le Fort II em vítima de trauma por acidente motociclístico: relato de caso
}

Surgical treatment of Le Fort I and Le Fort II fractures in victims of motorcycle accident trauma: case report Tratamiento quirúrgico de fracturas de Le Fort I y Le Fort II en víctima de accidente de motocicleta: reporte de caso Maria Eloise de Sá SIMON

Gustavo Antonio Correa MOMESSO

William Phillip Pereira da SILVA

Leonardo Alan DELANORA

Anderson Maikon de Sousa SANTOS

Tiburtino José de LIMA NETO

Leonardo Perez FAVERANI

Leonardo Costa de Almeida PAIVA

Faculdade de Odontologia de Araçatuba, Universidade Estadual Paulista, UNESP 16015-050 Araçatuba-SP, Brasil

\section{Resumo}

O terço médio da face é funcional e esteticamente importante. De acordo com a classificação Le Fort, existem três níveis mais fracos desta região da face quando traumatizados a partir de uma direção frontal, sendo que os acidentes motociclísticos, atualmente, correspondem a causa de aproximadamente $29 \%$ destes traumas. O presente trabalho tem como objetivo relatar um caso clínico de tratamento cirúrgico de fraturas do tipo Le Fort I e Le Fort II em um paciente de 29 anos de idade, sexo masculino, vítima de acidente motociclístico, atendido no Hospital de Emergência e Trauma Senador Humberto Lucena (João Pessoa - PB). Ao exame físico observou-se mobilidade de maxila, degrau palpável em pilar zigomático e pilar canino, alteração oclusal com leve mordida aberta e degrau em rebordo infraorbitário direito, entretanto o paciente não apresentava nenhuma alteração ocular. Foi solicitada tomografia computadorizada como exame complementar para confirmação do diagnóstico e planejamento cirúrgico, o qual se deu como fratura Le Fort I e Le Fort II no lado direito. O paciente foi submetido à cirurgia sob anestesia geral para fixação dos pilares zigomático e canino através do acesso vestibular maxilar e rebordo infraorbitário através do acesso subciliar. Inicialmente foi feito o bloqueio maxilo - mandibular para a utilização da oclusão como ponto de referência, seguido da redução das fraturas e fixação com placas e parafusos do sistema 2.0. Sob acompanhamento pós - operatório o paciente apresentou retorno da oclusão dentro dos padrões de normalidade, recuperou a projeção da região zigomática fraturada e então recebeu alta.

Descritores: Fraturas Ósseas; Fixação de Fratura; Traumatismos Faciais.

\section{Abstract}

The middle third of the face is functionally and aesthetically important. According to the Le Fort classification, there are three weaker levels of this region of the face when traumatized from a frontal direction, and motorcycle accidents currently account for approximately $29 \%$ of these traumas. This paper aims to report a clinical case of surgical treatment of Le Fort I and Le Fort II fractures in a 29-year-old male motorcycle accident victim treated at Senator Humberto Lucena Emergency and Trauma Hospital (João Pessoa - PB). Physical examination revealed maxillary mobility, palpable step in zygomatic and canine abutment, occlusal alteration with slight open bite, and right infraorbital ridge, but the patient had no ocular alteration. Computed tomography was requested as a complementary exam to confirm the diagnosis and surgical planning, which was the fracture of Le Fort I and Le Fort II on the right side. The patient underwent surgery under general anesthesia for fixation of the zygomatic and canine pillars through maxillary buccal access and infraorbital ridge through subciliary access. Initially, maxillary - mandibular blockade was performed to use the occlusion as a reference point, followed by fracture reduction and fixation with 2.0 system plates and screws. Under postoperative follow - up, the patient returned occlusion within the normal range, recovered the projection of the fractured zygomatic region and was discharged.

Descriptors: Bone Fractures; Fracture Fixation; Facial Injuries.

\section{Resumen}

El tercio medio de la cara es funcional y estéticamente importante. Según la clasificación de Le Fort, hay tres niveles más débiles de esta región de la cara cuando se traumatiza desde una dirección frontal, y los accidentes de motocicleta actualmente representan aproximadamente el $29 \%$ de estos traumas. Este artículo tiene como objetivo informar un caso clínico de tratamiento quirúrgico de fracturas de Le Fort I y Le Fort II en una víctima de accidente de motocicleta masculina de 29 años tratada en el Hospital de Emergencia y Trauma Senador Humberto Lucena (João Pessoa - PB). El examen físico reveló movilidad maxilar, paso palpable en el pilar cigomático y canino, alteración oclusal con leve mordida abierta y cresta infraorbitaria derecha, pero el paciente no tenía alteración ocular. Se solicitó la tomografía computarizada como un examen complementario para confirmar el diagnóstico y la planificación quirúrgica, que fue la fractura de Le Fort I y Le Fort II en el lado derecho. El paciente se sometió a cirugía bajo anestesia general para la fijación de los pilares cigomático y canino a través del acceso bucal maxilar y la cresta infraorbitaria a través del acceso subciliar. Inicialmente, se realizó un bloqueo maxilar mandibular para utilizar la oclusión como punto de referencia, seguido de la reducción y fijación de fracturas con placas y tornillos del sistema 2.0. Bajo seguimiento postoperatorio, el paciente regresó la oclusión dentro del rango normal, recuperó la proyección de la región cigomática fracturada y fue dado de alta.

Descriptores: Fracturas de Hueso; Fijación de Fracturas; Lesiones Faciales.

\section{INTRODUÇÃO}

O trauma afeta anualmente cerca de 5,8 milhões de pessoas, $32 \%$ a mais que a soma das mortes por malária, AIDS e tuberculose ${ }^{1}$, sendo a face frequentemente atingida devido à sua grande exposição.

Os acidentes automobilísticos, em especial os que envolvem motocicletas, são os principais agentes etiológicos desse tipo de fratura facial ${ }^{2,3}$, principalmente quando associado ao uso de álcool pelo condutor do veículo ${ }^{4}$.

O complexo do terço médio da face é constituído por uma série de pilares verticais os quais são suportados por pilares horizontais e que fornecem principalmente proteção contra forças direcionadas verticalmente. Este quadro resulta em alguns locais anatômicos de fraqueza, gerando padrões bastante previsíveis de fratura ${ }^{5}$.

Rene Le Fort no século XX definiu os três níveis mais fracos do complexo do terço médio da face quando traumatizados a partir de uma direção frontal, classificando os três padrões mais comuns em fraturas Le Fort I, Le Fort II e Le Fort III, sendo que 
cada padrão apresenta componentes característicos ${ }^{5}$.

O padrão de fratura Le Fort tipo I resulta de uma força direcionada acima dos dentes superiores, induzindo uma flutuação do palato. $\mathrm{O}$ exame da fratura Le Fort I deve ser feito segurando firmemente o arco superior com o dedo indicador posicionado por palatino e o polegar facialmente, tentando o deslocamento da maxila em três dimensões, bem como a compressão e expansão do arco maxilar. Maloclusão e mobilidade podem ser observadas ${ }^{5}$.

Já o padrão de fratura Le Fort tipo II, referido como fratura piramidal, decorre de uma força gerada no nível do osso nasal, ocasionando a mobilidade do terço médio da face através das órbitas e região média facial. $\mathrm{O}$ exame físico provavelmente revelará sinais perceptíveis da lesão. O edema recobrindo os locais fraturados normalmente está presente. $\mathrm{O}$ sinal de guaxinim clássico resultante de edema periorbital bilateral e equimoses pode ser observado ${ }^{5}$.

As possíveis complicações que podem acontecer no terço médio incluem, principalmente, parestesia de nervos, infecção, rinorréia do LCR, má oclusão, desvio de septo, problemas com fixação rígida, resultados estéticos ruins e ausência de união ou consolidação ${ }^{6}$.

\section{CASO CLÍNICO}

Paciente de 29 anos, sexo masculino, foi atendido no Hospital de Emergência e Trauma Senador Humberto Lucena (João Pessoa - PB) após sofrer acidente motociclístico. Ao exame clínico notou-se mobilidade de maxila, degrau palpável em pilar canino e pilar zigomático, alteração oclusal com leve mordida aberta e degrau em rebordo infraorbitário direito, sem apresentar alteração ocular (Figuras 1 e 2 ). Foi solicitado como exame complementar para confirmação do diagnóstico e planejamento cirúrgico a tomografia computadorizada, através da qual dignosticou-se fratura Le Fort I e Le Fort II do lado direito (Figura 3). Pelas condições apresentadas no exame clínico e de imagem, foi proposto tratamento cirúrgico aberto sob anestesia geral e intubação orotraqueal para fixação dos pilares zigomático e canino e do rebordo infraorbitário. Inicialmente foi feito o bloqueio maxilo - mandibular para a utilização da oclusão como ponto de referência, seguido da redução e fixação das fraturas em pilar canino e pilar zigomático com placas e parafusos do sistema 2.0, através do acesso vestibular maxilar. Logo após, foi também realizada a redução e fixação da fratura em rebordo infraorbitário direito com placas e parafusos do sistema 2.0, através do acesso subciliar (Figura 4). No pós-operatório de 45 dias foi possível notar um resultado satisfatório funcional e esteticamente com o retorno da oclusão aos padrões de normalidade e a projeção da região zigomática fraturada recuperada (Figuras 5 e 6 ).

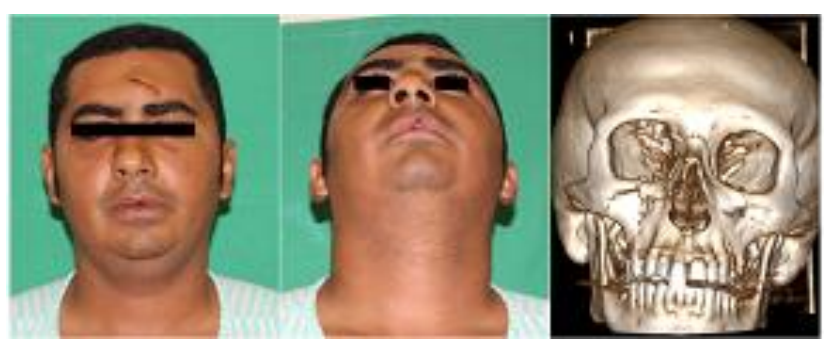

Figura 1: Pré-operatório. Ao exame clínico nota-se edema na região de terço médio devido as fraturas e exame de imagem com tomografia computadorizada em reconstrução 3D evidenciando fratura na região zigomática alterando sua projeção.

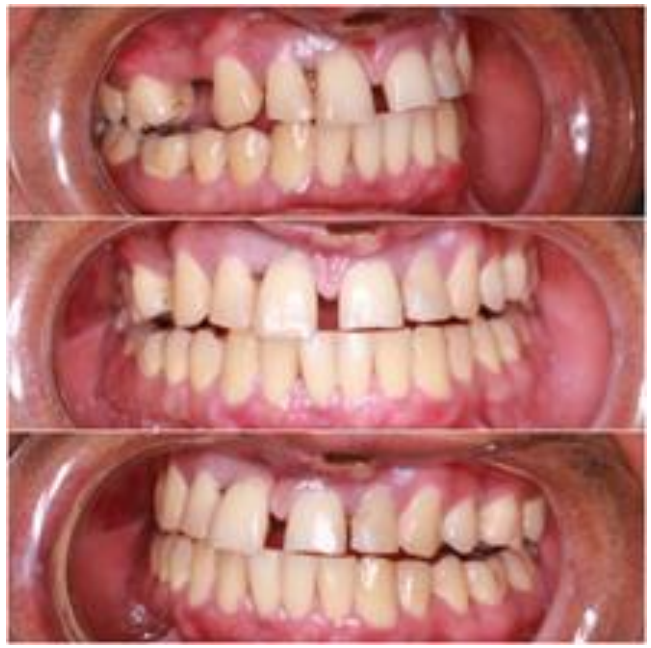

Figura 2: Pré-operatório. Paciente apresenta alteração oclusal com leve mordida aberta.

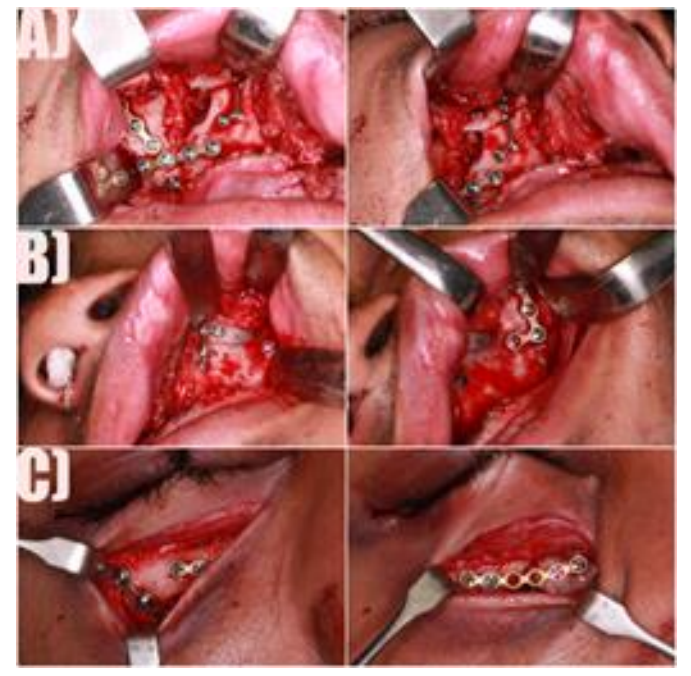

Figura 3: Trans-operatório - A e B)Fixação dos pilares canino e zigomático direito e esquerdo respectivamente, com placas e parafusos do sistema 2.0 através do acesso vestibular maxilar. C) Fixação de rebordo infraorbitário com placas e parafusos do sistema 2.0 através de acesso subciliar.

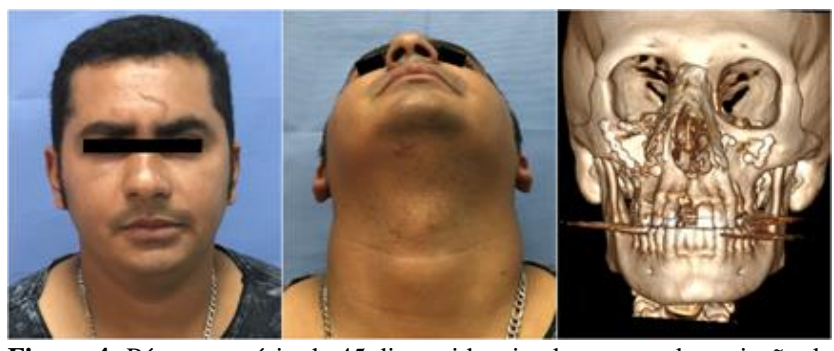

Figura 4: Pós- operatório de 45 dias evidenciando retorno da projeção da região zigomática devido a fixação das fraturas visíveis na tomografia computadorizada com reconstrução 3D. 


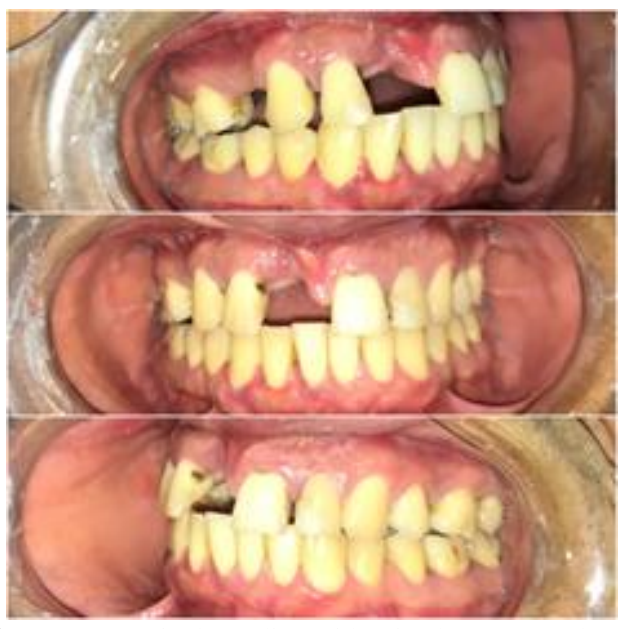

Figura 5: Pós-operatório de 45 dias evidenciando o retorno da oclusão do paciente aos seus padrões de normalidade.

DISCUSSÃO

Atualmente o trauma ainda afeta grande parte da população, sendo considerado um alarmante problema de saúde pública. O tratamento dessas lesões, especialmente na face, visa a recuperação tanto estética como funcional, com o mínimo de sequelas possíveis ao paciente ${ }^{6,7}$. Sendo assim, as fraturas em terço médio da face mostram-se um desafio à traumatologia bucomaxilofacial por ser uma região de relação funcional íntima com diversos sistemas vitais, além do importante significado estético, sendo essencial para o reconhecimento interpessoal e autoimagem ${ }^{5}$.

Os acidentes motociclísticos tem se mostrado o fator etiológico de maior ocorrência dentre as fraturas faciais ${ }^{8}$. Isto, somado ao aumento da velocidade dos veículos, torna a intensidade da força de impacto extremamente maior ${ }^{9}$, podendo levar a fraturas com o padrão descrito por Le Fort nas regiões de maior debilidade do esqueleto facial ${ }^{10}$.

Porém, no trauma dificilmente as forças de impacto são perfeitamente localizadas, o que pode resultar em combinações imprevisíveis das fraturas, como no caso apresentado, no qual o paciente apresentou fraturas Le Fort I e Le Fort II em um dos lados 5 .

Em caso de fratura Le Fort I, a maloclusão está entre as principais complicações que podem atingir o paciente ${ }^{11}$. Dessa forma, é necessária a realização adequada da técnica cirúrgica da redução e fixação nos pilares caninos e zigomáticos para que possa ser alcançado o restabelecimento tanto estético como funcional ${ }^{12}$.

Ademais, o tempo decorrido após o trauma é um fator de essencial importância no direcionamento do tratamento das fraturas faciais. Este período deve ser o mais breve possível, priorizando o atendimento imediato, pois dentro de poucos dias a fratura não tratada pode sofrer complicações devido a formação de fibroses e início da consolidação do tecido ósseo ${ }^{13}$, fato corroborado pelo caso apresentado já que o tratamento foi realizado no período adequado.

\section{CONCLUSÃO}

As fraturas Le Fort I e Le Fort II podem ocorrer em indivíduos submetidos a traumas de grande impacto em direção anterior, como nos casos de acidentes motociclísticos. No tratamento destas fraturas, a fixação dos pilares caninos e zigomáticos tem mostrado sucesso na obtenção de um bom resultado estético e funcional ao paciente, colaborando para o retorno da oclusão aos padrões de normalidade.

\section{REFERÊNCIAS}

1. OBUBR. Organização das Nações Unidas no Brasil. Traumas matam mais que malária, tuberculose e AIDS, alerta OMS. Disponível em: $<$ http://www.onu.org.br/traumas-matam-maisque-malaria-tuberculose-e-aids-alerta-oms/>. Acesso em: 22 julho 2019

2. Ansari MH. Maxillofacial fractures in Hamedan province, Iran: a retrospective study (1987-2001). J Craniomaxillofac Surg. 2004;32(1):28-34.

3. Kostakis G, Stathopoulos P, Dais P, Gkinis G, Igoumenakis D, Mezitis M, Rallis G. An epidemiologic analysis of 1,142 maxillofacial fractures and concomitant injuries. Oral Surg Oral Med Oral Pathol Oral Radiol. 2012; 114(5 Suppl):S69-73.

4. Li Z, Li ZB. Characteristic changes of pediatric maxillofacial fractures in China during the past 20 years. J Oral Maxillofac Surg 2008;66:2239-42.

5. Fonseca RJ. Trauma Bucomaxilofacial 4. ed. Rio de Janeiro : Elsevier; 2015.

6. Wulkan M, Parreira Junior JG, Botter DA. Epidemiologia do trauma facial. Rev Assoc Med Bras. 2005;51(5):290-95.

7. Scherer M, Sullivan WG, Smith DJ Jr, Phillips LG, Robson MC. An analysis of 1,423 facial fractures in 788 patients at an urban trauma center. J Trauma. 1989;29(3):388-90.

8. Cohen RS, Pacios AR. Facial and cranio-facial trauma: epidemiology, experience and treatment. F Med. 1995;111(suppl):111-16.

9. de Birolini D, Utiyama E, Steinman E. Cirurgia de Emergência. São Paulo: Atheneu; 1997.

10. Tessier P. The classic reprint: experimental study of fractures of the upper jaw. 3. René Le Fort, M.D., Lille, France. Plast Reconstr Surg. 1972;50(6):600-7.

11. Buehler JA, Tannyhill RJ 3rd. Complications in the treatment of midfacial fractures. Oral Maxillofac Surg Clin North Am. 2003; 15(2):195-212.

12. Manson PN, Clark N, Robertson B, Slezak S, Wheatly M, Vander Kolk C, Iliff N. Subunit principles in midface fractures: the importance of sagittal buttresses, soft-tissue reductions, and sequencing treatment of segmental fractures. Plast Reconstr Surg. 1999;103(4):1287-306. 
13. Carr RM, Mathog RH. Early and delayed repair of orbitozygomatic complex fractures. J Oral Maxillofac Surg. 1997;55(3):253-8; 258-9.

\section{CONFLITO DE INTERESSES}

Os autores declaram não haver conflitos de interesse.

\section{AUTOR PARA CORRESPONDÊNCIA}

\section{Maria Eloise de Sá Simon}

Faculdade de Odontologia de Araçatuba, UNESP

16015-050 Araçatuba-SP, Brasil

E-mail: eloise.simon@unesp.br 